\title{
Fish tank granuloma
}

\author{
S F Gray, R Stanwell Smith, N J Reynolds, E W Williams
}

A history of tropical fish keeping should be sough in patients with unusual or persistent skin lesions of the hands and fingers
Control of Infection Service, Bristol BS1 3NP $\mathrm{S} F$ Gray, MRCP, registrar in public health medicine

R Stanwell Smith, MFCM, consultant in public health medicine

Bristol Royal Infirmary, Bristol BS2 8HW N J Reynolds, MRCP, registrar in dermatology E W Williams, MRCPATH, consultant microbiologist

Correspondence to: Dr Williams.

BrMed F 1990;300:1069-70
Mycobacterium marinum is an opportunist pathogen commonly found in aquatic environments. Infected fish may develop a disseminated granulomatous disease - fish tuberculosis - that leads to wasting and ultimately to death. In humans this organism causes local infection after contamination of open wounds or abrasions. $M$ marinum skin infection acquired from tropical fish tanks was first described in 1962 by Swift and Cohen, ${ }^{1}$ and has since been reported by many workers world wide. ${ }^{2} M$ marinum is the causative organism in swimming pool granuloma, which is well known in the United Kingdom ${ }^{3}$; infections of humans with $M$ marinum after fishing, boating, and other exposure to salt and brackish water has also been described. $^{4-7}$ We report on four patients who were diagnosed as having $M$ marinum infection causing tropical fish tank granuloma over eight months in 1988. There had been no other cases locally in the previous five years. Epidemiologically there was no link among the cases.

\section{Case reports}

Case 1-A 36 year old shop fitter cut his thumb while cleaning his fish tank. The wound failed to heal despite oral flucloxacillin and over the next 12 months developed into a painful red plaque (fig 1 ) that intermittently discharged pus. After referral to a dermatologist tropical fish tank granuloma was diagnosed clinically, and although acid fast bacilli were not seen on microscopy, $M$ marinum was grown from the biopsy material. The lesion improved rapidly after a six week course of co-trimoxazole consisting of two tablets twice a day.

Case 2-A 48 year old unemployed man cut his right



FIG $1-M$ marinum granuloma hand on some glass. The cut did not heal, and over the next two weeks he developed a painful red plaque at the puncture site. This was surgically incised, and he was given intravenous flucloxacillin and penicillin. Over the next four days a further six large painful erythematous nodules appeared, extending progressively up his arm. Biopsy material subsequently showed granulomas with no caseation and acid fast bacilli, and $M$ marinum was isolated. Treatment with co-trimoxazole two tablets twice a day was started, but because the inflammation of one nodule increased minocycline 100 mg twice a day was added after one week. All lesions were flat and not tender after 14 weeks of treatment.

Case 3-A 48 year old teacher sustained a trivial cut to his right middle finger just before cleaning out his fish tank. The wound failed to heal and over the next two weeks developed into a painful red lesion. A second red lesion measuring $2 \mathrm{~cm} \times 2 \mathrm{~cm}$ subsequently appeared on the back of his hand and intermittently discharged purulent fluid. There was no improvement despite a week's course of flucloxacillin and a further course of erythromycin, and the lesions were subsequently lanced in a local accident and emergency department. Over the next three weeks he developed a further 15 to 20 painful discharging nodules that spread proximally from his right hand to upper arm (fig 2). Two months after the initial injury referral to a dermatologist resulted in a clinical diagnosis of $M$ marinum infection, which was confirmed by microscopy and culture of biopsy material. Treatment with rifampicin $600 \mathrm{mg}$ and isoniazid $300 \mathrm{mg}$ daily was started. After completing four months of treatment the nodules were healing but still visible.

Case 4-A 22 year old zoo keeper who worked in the aquarium and reptile house noticed a small cut on the back of his hand, which failed to heal and developed into a painful purplish plaque measuring $2 \mathrm{~cm} \times 2 \mathrm{~cm}$. A second, larger lesion developed two months later on his forearm. There was no improvement after five months despite two courses of erythromycin prescribed by his general practitioner. Referral to a dermatologist led to a clinical diagnosis of $M$ marinum infection, which was later confirmed by culture of biopsy material, though no acid fast bacilli were seen on microscopy. A three month course of co-trimoxazole three tablets twice a day was started with clinical improvement after two months.

\section{EPIDEMIOLOGICAL SURVEY}

Each patient was visited at home to investigate the type of tank and fish, to inquire about new stock and fish illness, and to collect water specimens and tank swabs. All four men had acquired new fish shortly before their injury; in three of the four cases the new stock had died over the next few weeks or months. The new stock included a variety of species of tropical fish from four different suppliers and included some that had been directly imported from Singapore. The shopkeepers were unaware of a problem of fish tuberculosis in their stock, although two commented on an association with Malawian cichlids (the species acquired by case 1) some years previously. The zoo keeper recognised that fish were dying of tuberculosis, but he continued to put his injured hand into the tank and to 


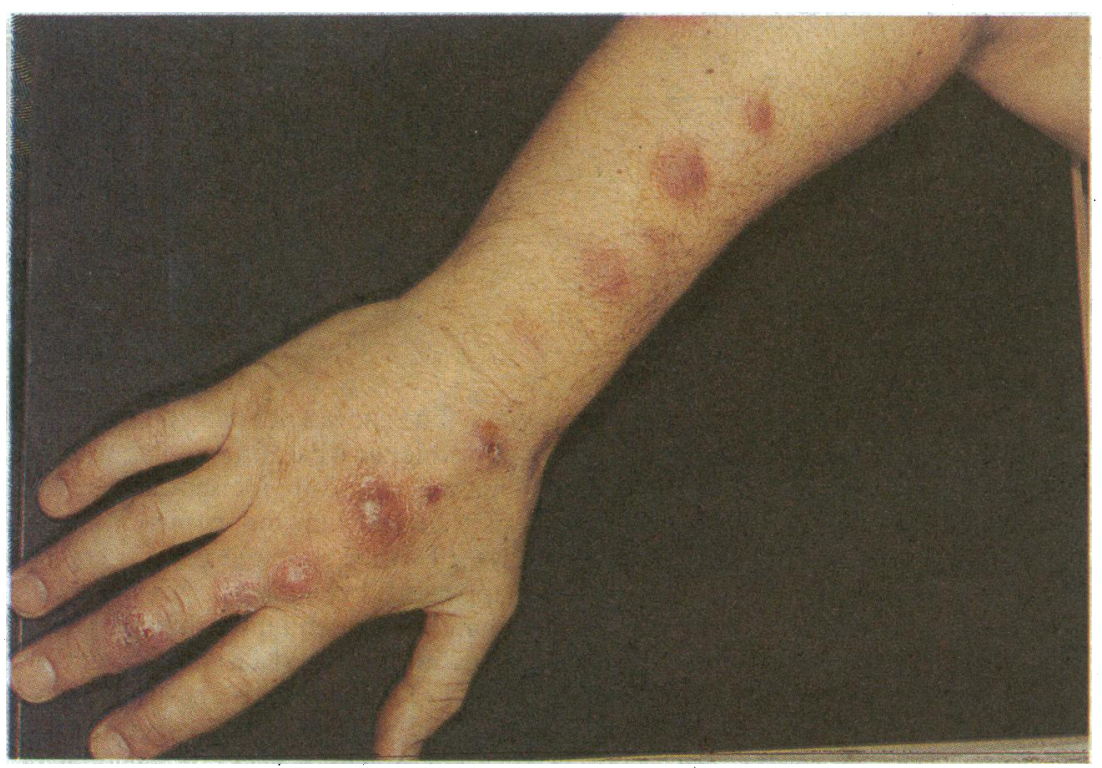

FIG 2-Sporotrichoid form of $M$ marinum granuloma

handle dead fish without gloves. None of the men were aware that skin infection could be acquired from fish tanks. The tanks were well maintained. None of the water specimens or tank swabs grew $M$ marinum, but all were taken several months after the infection was diagnosed in the four men.

We sent members of three tropical fish clubs in Bristol a questionnaire to determine their knowledge and experience of fish tuberculosis. The 40 respondents had kept fish for between one and 60 years; 34 immersed their hands at least once a week in their tanks, and only two ever wore gloves. Many were familiar with fish "wasting disease": 24 reported that they would probably or definitely recognise fish tuberculosis; five of this group had diagnosed this disease in their fish in the past year, and 13 believed it had caused the death of fish at some time in the past. Less than a third (12) of the respondents, however, were aware of the risk of skin infections after contact with fish. Only 13 of the fish keepers covered cuts or took care not to immerse a cut hand in the tank, and 30 reported that they would handle dead fish with their hands. In the past 12 months two of the respondents had noticed cuts on their hands that had taken more than two months to heal or unusual skin lumps that had lasted more than two months.

\section{Discussion}

Although well known to dermatologists, infection with $M$ marinum is less well recognised by nonspecialists, and there may be a substantial delay between the appearance of lesions and the correct diagnosis. This occurred in our patients. The usual presentation of the skin infection is as a solitary granulomatous nodule or pustule, which may ulcerate and discharge or form a suppurative mass. There are often multiple lesions, and it is not uncommon for one or more nodules to extend along the line of lymphatic vessels (sporotrichoid form). Immunocompromised people may develop disseminated skin lesions. Tenosynovitis has been reported after penetrating injury to the hand by fish spines. ${ }^{8}$

The diagnosis is confirmed by the growth of $M$ marinum in cultures of biopsy material from the skin lesions. This may take several weeks. Acid fast bacilli are often present in small numbers and may not be detected by microscopy. A special culture procedure is required. The biopsy specimens from our four patients were cultured on Löwenstein-Jensen medium at room temperature, $30^{\circ} \mathrm{C}$, and $37^{\circ} \mathrm{C}$. The cultures grown at room temperature and $30^{\circ} \mathrm{C}$ yielded typical pigmented mycobacterial colonies in two to four weeks. There was no growth at $37^{\circ} \mathrm{C}$, the usual temperature used for culturing mycobacteria. Tests to determine full identification and sensitivity take a further few weeks.

Although spontaneous resolution can occur, healing may take several months. ${ }^{9}$ Huminer et al reviewed the treatment of the infection. ${ }^{2}$ Several chemotherapeutic regimens have proved effective and include cotrimoxazole, ${ }^{9}$ high doses of minocycline, and combinations of antituberculous drugs. Unfortunately, no control trials have been performed and results of in vitro sensitivity tests may not accurately reflect the in vivo response to treatment. There are reports of an initial poor response to one drug or combination of drugs with a subsequently good response when treatment was switched to another regimen. ${ }^{10}$ The role of surgery is controversial, and surgical incision or exploration may lead to the spread of infection into deeper tissues with the subsequent development of tenosynovitis ${ }^{11}$ or bursitis. ${ }^{12}$ Rapid sporotrichoid spread occurred shortly after a surgical procedure in two of our cases (cases 2 and 3).

Fish tuberculosis is a chronic infection. There is no effective treatment, but as it may take up to 12 months to cause death it is not perceived as a problem by breeders or dealers. $M$ marinum survives readily in water and can be cultured from dead fish, the sides of tanks, water samples, and sand samples. Identification of acid fast bacilli in a homogenate of dead fish has been used to confirm a clinical diagnosis of $M$ marinum infection in humans at an early stage. ${ }^{9}$ Though the fish fanciers in our survey commonly reported recent or past fish tuberculosis in their stock, neither they nor our four patients were aware that this skin condition could be acquired from their hobby. Simple preventive measures, such as covering cuts and grazes on hands, should be recommended to fish handlers. Unusual or persistent skin lesions on hands or fingers should prompt doctors to inquire about the keeping of tropical fish. This hobby is increasing in popularity, and it is important to improve clinical awareness of the disease so that it can be diagnosed at an early stage and managed appropriately.

We thank the following consultant dermatologists from Bristol Royal Infirmary for permission to report the cases: Dr R R M Harman, Dr C T C Kennedy, and Dr R D G Peachey. We thank Dr P A Jenkins, Mycobacterium Reference Laboratory, Cardiff, for help and encouragement.

1 Swift S, Cohen H. Granulomas of the skin due to Mycobacterium balnei after abrasions from a fish tank. N Engl F Med 1962;267:1244-6.

2 Huminer D, Pitlik SD, Block C, Kaufman L, Amit S, Rosenfeld JB. Aquarium-borne Mycobacterium marinum skin infection. Report of a cas and review of the literature. Arch Dermatol 1986;122:698-703.

3 Galbraith NS, Barrett NJ, Stanwell-Smith R. Water and disease after Croydon: a review of water-borne and water associated disease in the UK 1937-1986. Fournal of the Institution of Water and Environmental Managemen 1987;1:7-21.

4 Collins $\mathrm{CH}$, Grange JM, Noble WC, Yates MD. Mycobacterium marinum infections to man. Fournal of Hygiene 1985;94:135-49.

5 Johnston JM, Izumi AK. Cútaneous Mycobacterium marinum infection ("swim-

ming pool granuloma"). Clin Dermatol 1987;5:68-75.

6 Zeligman I. Mycobacterium marinum granuloma. A disease acquired in the tributaries of Chesapeake Bay. Arch Dermatol 1972;106:26-31.

7 Jolly HW, Seabury JH. Infections with Mycobacterium marinum. Arch Dermatol 1972;106:32-6.

8 Wagner RF, Tawil AB, Colletta AJ, Hurst LC, Yecies LD. Mycobacterium marinum tenosynovitis in a Long Island fisherman. NY State $7 \mathrm{Med}$ 1981;81:1091-4.

9 Black MM, Eykyn SJ. The successful treatment of tropical fish tank granuloma (Mycobacterium marinum) with co-trimoxazole. $\mathrm{Br}$ f Dermato 1977;97:689-92.

10 Wallace RJ. Recent clinical advances in knowledge of the nonleprous environmental mycobacteria responsible for cutaneous disease. Arch Dermatol 1987;123:337-9.

11 Hay RL, McCarthy OR, Marks J. Fish tank granuloma. Br Med f 1975;i:268.

12 Winter FE, Runyon EH. Prepatellar bursitis caused by Mycobacterium marinum (balnei). Case report, classification, and review of the literature. f Bone foint Surg [Am] 1965;47A:375-9.

(Accepted 4 October 1989) 